\title{
A strange case of cervical pain
}

\author{
Jonathan Montomoli, Davide Lazzarini", Luca Morolli, Giorgio Ioli
}

Third Department of Medicine, Rimini, Italy; ${ }^{*}$ Corresponding Author: davide.lazzarini@auslrn.net

Received 29 September 2011; revised 21 October 2011; accepted 15 November 2011

\begin{abstract}
A 56-year-old man was admitted to hospital with fever, progressive fatigue, bilateral shoulder pain, paresthesias and stiffness after awakening. Differential diagnosis were several diseases such as rheumatoid arthritis, fibromyalgia and infectious spondylitis. Routine laboratory assays showed mild anemia and increased markers of inflammation. The worsening of clinical conditions led to performing a Computerized Tomography and a Cervical Magnetic Resonance Imaging which demonstrated an epidural liquid area in both left and anterior section of the spinal canal. Due to the high suspicious of tuberculosis we started a therapy with isoniazid, rifampicin, pyrazinamide and cyprofloxacin, even if the results of culture was negative for any kind of bacteria. This case-report underlines the difficulties of a correct diagnosis in a common symptom as back pain is.
\end{abstract}

Keywords: Inflammatory Back Pain; Spinal Tuberculosis; Fibromyalgia

\section{INTRODUCTION}

Inflammatory back pain (IBP) is a frequent cause that leads to medical attention and is defined by clinical features as morning stiffness with more than 30 minutes' duration, improvement with exercise but not with rest, persistence for at least 3 months, age less than 50 years, etc. [1,2]. Althoughit is a typical presentation of the seronegative spondylarthropathies, its differential diagnosis included several diseases such as rheumatoid arthritis, fibromyalgia and infectious spondylitis.

Tuberculosis is one of the most common infectious diseases in the world and its incidence is increasing in the western countries since the early 1900s [3]. Extrapulmonary forms account for $10 \%-40 \%$ of all cases of tuberculosis [4] and approximately $50 \%$ of cases of skeletal tuberculosis occur in the spine [5]. When it occurs the onset of the symptoms is insidious and some of the clinical findings may be fulfill IBP criteria. Moreover any delay in the diagnosis increases the probability of functional sequelae $[4,6]$.

\section{CASE REPORT}

A 56-year-old man was admitted to our department because of dull cervical pain which had worsened over the previous month. Pain was radiated to the bilateral shoulder regions and upper extremities, associated with paresthesias and stiffness for 3 - 4 hours after wakening that improved with movement. He also reported intermittent fever. Routinary chest-X-ray showed calcified nodules in the median right lung field and in the perihilar region on the same side. No significant medical history except chronic cervical pain since years and hypertension treated by amlodipin. A cervical-x-ray revealed narrowing of the disc space between $5^{\text {th }}$ cervical and $1^{\text {st }}$ thoracic vertebral bodies.

The patient had been evaluated for cervical pain one month before and based on physical examination was suspected rheumatic polymyalgia. For this reason he started prednisolone $125 \mathrm{mg} /$ day per os and follow-up. He did not attend the check-up fixed after 10 days.

On physical examination was noted cervical stiffness associated with slight hyposthenia of the left arm and right leg. Babinski's sign was absent bilaterally. Blood pressure was 150/80 $\mathrm{mmHg}$ and pulse rate was regular, $60 \mathrm{bpm}$. Routine laboratory assays reported in Table 1 showed mild anemia and increased markers of inflammation as erythrocyte sedimentation rate (ESR), fibrinogen, alpha-1-acid glycoprotein and C-reactive protein (CRP). No monoclonal peak was detected in serum protein electrophoresis and Bence-Jones protein was absent. Two days after admission the patient presented worsening of clinical conditions with comparison of moderate motor deficit characterized by hypostenia of the left arm and right leg. Hypertonus associated with brisk deep tendinous reflexes were presented on the right leg. Babinski's sign was present on the right. Was performed a Computerized Tomography (CT) of the thorax which confirmed the calcified lung nodules on upper part of the inferior lob of the right lung. Further calcified nodules were revealed at the base of the left lung. Some lymph nodes located caudally to the carina of the trachea were 
Table 1. Blood test screening upon admission.

\begin{tabular}{lcc}
\hline & & Reference value \\
\hline WBC (10/) & 6.57 & $4.30-10.00$ \\
Neu (\%) & 76.9 & $40.0-75.0$ \\
Lyn (\%) & 16.0 & $19.0-48.0$ \\
Mon (\%) & 6.2 & $1.0-10.0$ \\
Eos (\%) & 0.6 & $0-6.0$ \\
Bas (\%) & 0.3 & $0-1.5$ \\
RBC (10 $\left.{ }^{12} / \mathbf{l}\right)$ & 3.86 & $4.40-6.00$ \\
Hb (g/d) & 11.2 & $14.0-18.0$ \\
Hct (\%) & 32.6 & $41.0-51.0$ \\
MCV (fl) & 84 & $80-96$ \\
MCH (pg) & 29.0 & $27.0-34.0$ \\
MCHC (g/dl) & 34.3 & $32.0-36.0$ \\
PLT (10 $\left.{ }^{9} / \mathbf{l}\right)$ & 324 & $140-440$ \\
C-reactive protein CRP (mg/l) & 12.3 & $<5$ \\
ESR mm/h & 80 & $<15$ \\
Fibrinogen (mg/dl) & 445 & $200-400$ \\
Alfa1-glycoprotein (mg/d) & 146 & $30.0-135.0$ \\
\hline
\end{tabular}

slightly enlarged. Cervical magnetic resonance imaging (MRI) revealed evidence of spondylodiscytes involving C3, C4, and C5 vertebral bodies and the respective intervertebral disks. An epidural liquid area compatible with abscess occupied the left and anterior section of the spinal canal at the same level determining moderate posterior and right deviation of the spinal cord. A smaller abscess was present at the level of the intervertebral space D9-D10 without any compression on the spinal cord. Nothing was noticed from cerebral MRI. Percutaneous biopsy of the lesions located at the level C3-C4 was performed using CT guidance. The result of the culture was negative for $\mathrm{M}$. tuberculosis or other bacteria. Due to the high suspicious of tuberculosis as soon as was obtained the MRI result therapy with isoniazid, rifampin, pyrazinamide and cyprofloxacin was started. MRI performed 10 days after therapy start revealed mild decrease of the abscess dimension. Patients was discharged one month after admission. Therapy was continued for 9 months and patients recovered completely from neurological deficits. No relapse of the infection after 2 years.

\section{DISCUSSION}

We considering this case report an interesting example of how high degree of suspicious is required in spinal tubercolosis diagnosis [6]. The patient had some troubles in his life and at work; he was depressed and he attributed all symptoms to stress. When he was evaluated for the first time, symptoms partially fulfilled IBP criteria and polymialgia was suspected, even if it is a diagnosis of exclusion [7]. Corticosteoroids probably accelerated spread of infection and comparison of neurological deficits. Appearance of motor deficits led to perform rapidly further diagnostic exams of imaging that revealed vertebral abscess due to infection. The incidence of neurological deficits at the time of diagnosis in patients with spinal tuberculosis is reported to be $20 \%$ to $76 \%$ [4]: these data suggest that spinal tuberculosis is often not considered as responsible of IBP until comparison of neurological signs that are related to compression of spinal cord [8]. Fever, that is still considered an important sign of infection, it is reported being absent in almost two-third of cases [4], contributed to delay the correct diagnosis.

Moreover, laboratory data generally show mild anemia and the presence of a raised ESR and high levels of C-reactive protein [9] suggested that an inflammatory cause supported spinal pain. However, similar laboratory results are obtained also in patients with polymialgia or other rheumatic disease. About imaging, radiography is rarely useful into detection of spinal tuberculosis but chest-X-ray could show lung calcifications related to former infection sustained by M. Tubercolosis and that could orientate physician to consider the possibility of a relapse of infection with extra pulmonary localization. Differently, both MRI and CT are usually determinant for diagnosis as happened in this case. In general, MRI is more sensitive in the early stages of the disease and CT allows better visualization of the spinal canal. Lastly, percutaneous biopsy, even if CT-guided, didn't isolate the pathogen. Colmenero et al. reported positive culture of the percutaneous bone biopsy in $48 \%$ of tubercolosis vertebral osteomyelitis [4].

\section{REFERENCES}

[1] Rudwaleit, M., Metter, A., Listing, J., Sieper, J. and Braun, J. (2006) Inflammatory back pain in ankylosing spondylitis: A reassessment of the clinical history for application as classification and diagnostic criteria. Arthritis \& Rheumatism, 54, 569-578. doi:10.1002/art.21619

[2] Calin, A., Porta, J., Fries, J.F. and Schurman, D.J. (1997) Clinical history as a screening test for ankylosing spondylitis, The Journal of the American Medical Association, 237, 2613-2614. doi:10.1001/jama.1977.03270510035017

[3] Barnes, P.F. and Barrows, S.A. (1993) Tuberculosis in the 1990s. Annals of Internal Medicine, 119, 400-410.

[4] Colmenero, J.D., Jimenez-Mejias, M.E., Reguera, J.M., et al. (2004) Tuberculous vertebral osteomyelitis in the new millennium: Still a diagnostic and therapeutic challenge. European Journal of Clinical Microbiology \& Infectious Disease, 23, 477-483. doi:10.1007/s10096-004-1148-y 
[5] Cantini, F., Salvarani, C., Olivieri, I., et al. (1998) Tuberculous spondylitis as a cause of inflammatory spinal pain: A report of 4 cases. Clinical and Experimental Rheumatology, 16, 305-308.

[6] Wierzba-Bobrowicz, T, et al. (2010) Case report. Cervical spinal tuberculosis. Folia Neuropathologica, 48, 300-304.

[7] Ceccato, F., et al. (2011) Conditions mimicking polymy- algia rheumatica. Reumatologia Clinica, 7, 156-160.

[8] Ostelo, R.W., et al. (2008) Interpreting change scores for pain and functional status in low back pain: Towards international consensus regarding minimal important change. Spine, 33, 90-94.

[9] Amin, I., et al. (2011) PCR could be a method of choice for identification of both pulmonary and extra-pulmonary tuberculosis. BMC Research Notes, 4, 332. 